\title{
„Heimat“ neu denken? „Heimat“ als umkämpfter Begriff im österreichischen Bundespräsidentschaftswahlkampf 2016
}

\author{
Andrea Tony Hermann ${ }^{1,2}$ \\ 1 Institut für Strategieanalysen, Wien \\ 2 die siebente fakultät: Zentrum für Gesellschaft, Wissen und Kommunikation, Karl-Franzens-Universität Graz \\ andrea.hermann@strategieanalysen.at
}

\section{Zusammenfassung}

Der Begriff „Heimat“ wurde in Österreich lange insbesondere durch rechte und konservative PolitikerInnen besetzt. Mittlerweile ist der Begriff zunehmend umkämpft, da PolitikerInnen anderer Parteien versuchen, ihn mit alternativen Bedeutungen zu füllen. Vor diesem Hintergrund werden die Deutungsangebote für "Heimat“ im österreichischen Bundespräsidentschaftswahlkampf 2016 analysiert. Der Artikel greift hierfür auf diskurstheoretische Überlegungen und insbesondere Laclaus Konzept der „leeren Signifikanten“ zurück. Er rekonstruiert die Heimatverständnisse der Stichwahlkandidaten Van der Bellen und Hofer entlang ihrer räumlichen, zeitlichen, thematischen, normativen, emotionalen und symbolischen Bezüge sowie der dadurch bereitgestellten Identitätsangebote. Abschließend diskutiert der Artikel die Erkenntnisse in Bezug auf die Forschungsliteratur und mögliche (wahlkampf-)strategische Erwägungen.

\section{Schlüsselwörter}

Bundespräsidentschaftswahl 20I6, Diskursanalyse, Heimat, Österreich, politisches Konzept

\section{Rethinking „Heimat"? „Heimat“ as a Contested Term in the Austrian Presidential Election Campaign of 2016}

\begin{abstract}
In Austria, the term "Heimat" has primarily been used by right-wing and conservative politicians for a long time. Nowadays the term becomes increasingly contested because politicians of other parties attempt to assign different meanings to it. Against this background, the article analyzes the interpretations of "Heimat" during the Austrian Presidential Election Campaign of 2016. Therefore, it draws on discourse theoretical considerations and particularly Laclau's concept of "empty signifiers". The different understandings of "Heimat" of the second ballot candidates Van der Bellen and Hofer are reconstructed along their geographical, temporal, topical, normative, emotional and symbolic references and the thereby provided concepts of identity. The article concludes by discussing its findings regarding scholarlyworks on the term "Heimat" and potential(election) strategic considerations.
\end{abstract}

\section{Keywords}

Austria, discourse analysis, Heimat/home, political concept, presidential elections 2016

The author has declared that no competing interests exist. 


\section{Einführung}

Nicht erst vor dem Hintergrund von Migrationsbewegungen, der Europäisierung von Politikfeldern und dem Aufstieg des Rechtspopulismus spielt „Heimat“ in öffentlichen Diskursen im deutschsprachigen Raum eine Rolle (Kühne/Schönwald 2015). In Österreich besetzten lange Zeit vor allem konservative und rechte PolitikerInnen und Parteien, insbesondere die Freiheitliche Partei Österreichs (FPÖ), den Begriff (Kühne/Spellerberg 20IO; Kühne 2OII; Bastian I995; Marquart 2OI3; Preglau 200I). Die sich selbst als „Soziale Heimatpartei“ (Freiheitliche Partei Österreichs 20I9) bezeichnende FPÖ bedient sich seit den I990er Jahren regelmäßig und verstärkt seit Mitte der 20ooer Jahre des Begriffs auf Plakaten und bei Slogans (Marquart 20I3). Sie titelte beispielsweise „Heimat statt Schüssel und Brüssel“ im Nationalratswahlkampf 2006 (Demokratiezentrum Wien 2019) oder „Heimatliebe statt Marokkaner-Diebe“ bei der Innsbrucker Kommunalwahl 2012 (Marquart 20I3).

In den letzten Jahren scheint der Begriff „Heimat“ jedoch zunehmend umkämpft zu sein: Im Frühjahr 2018 präsentierte Michael Ludwig „Heimat“ als eines von vier zentralen Handlungsfeldern, die er in der ersten Plakatkampagne als neuer Wiener Gemeinde- und Landesorganisationsvorsitzender der Sozialdemokratischen Partei Österreichs (SPÖ) und Wiener Bürgermeister vorstellte (Gaigg 20I8; Stuhlpfarrer 20I8). Bereits 2016 avancierte „Heimat“ im Rahmen der Stichwahl im österreichischen Bundespräsidentenwahlkampf zu einem wichtigen Motiv des ehemals grünen Parteiobmanns Alexander Van der Bellen und wurde von ihm und seinem Mitbewerber Norbert Hofer häufig thematisiert (Bernhardt/Liebhart 20I7; Liebhart/Bernhardt 20I7).

Gerade Bundespräsidentschaftswahlkämpfe sind aufgrund der Rolle des Bundespräsidenten für die Analyse des Heimatbegriffs in politischen Kontexten interessant. Aufgrund der starken Legitimation durch die Direktwahl, des üblichen Rollenverzichts und der überparteilichen Amtsführung verstehen sich Bundespräsidenten traditionell als Repräsentanten aller ÖsterreicherInnen (Pelinka/Rosenberger 2007; Pelinka 2004). Entsprechend werden in den Wahlkämpfen um das Amt oftmals grundlegende gesellschaftliche Werte und Vorstellungen verhandelt und somit auch das Verständnis dessen, wie Österreich und seine BürgerInnen nach innen und außen repräsentiert werden sollen. In diesem Kontext kann „Heimat“ eine symbolische Funktion für die Vorstellungen der Präsidentschaftskandidaten von Österreich zukommen und der Begriff daher eine bedeutendere Rolle spielen als beispielsweise bei Nationalratswahlkämpfen, in denen tagesaktuelle Themen meist wichtiger sind.

Die spezifischen parteipolitischen Hintergründe der Stichwahlkandidaten für das Amt des Bundespräsidenten im Jahr 20I6, mit denen sehr unterschiedliche gesell- schaftspolitische Positionen einhergehen, ließen eine besondere Umkämpftheit des Begriffs erwarten. Während Alexander Van der Bellen als unabhängiger Kandidat und ehemaliger Bundesparteiobmann der Grünen Partei für das links-liberale Lager stand, repräsentierte Norbert Hofer als Kandidat der Freiheitlichen Partei Österreichs das rechtspopulistische Lager. Diese Polarisierung lässt Verständnisse von „Heimat“ vermuten, die unterschiedlicher sind als in einem Stichwahlkampf zwischen Kandidaten, deren Parteien sich eher in der gesellschaftspolitischen Mitte verorten. Stichwahlkandidaten der Österreichischen Volkspartei (ÖVP) und der Sozialdemokratischen Partei Österreichs (SPÖ) würden wahrscheinlich ähnlichere Heimatbegriffe repräsentieren.

Vor diesem Hintergrund untersucht dieser Artikel die Deutungsangebote für „Heimat“ im österreichischen Bundespräsidentschaftswahlkampf 20I6. Er zeigt, dass sich in den Kampagnen der beiden Kandidaten sehr unterschiedliche Verständnisse des Heimatbegriffs gegenüberstehen, die sich insbesondere durch den Grad der Offenheit und Veränderbarkeit unterscheiden. Nach einer konzeptionellen Annäherung an den Begriff (siehe Kapitel 2) erläutert der Artikel die ihm zugrundeliegenden diskurstheoretischen Überlegungen (siehe Kapitel 3). Zur Umsetzung dieser stützt er sich auf eine multimodale Diskursanalyse von Video- und Interviewtranskripten sowie Wahlplakaten der Stichwahlkandidaten Alexander Van der Bellen und Norbert Hofer, die sprachliche und visuelle Methoden kombiniert (siehe Kapitel 4). Der Artikel rekonstruiert die Heimatverständnisse der Kandidaten illustrativ entlang ihrer räumlichen, zeitlichen, thematischen, normativen, emotionalen und symbolischen Bezüge sowie der dadurch bereitgestellten Identitätsangebote (siehe Kapitel 5). Van der Bellen repräsentiert einen offenen, dynamischen und integrativen Heimatbegriff, der trotzdem auf ein idealtypisches, ländlich geprägtes Heimatbild rekurriert. Hofer hingegen vertritt ein eher geschlossenes, statisches und exklusives Verständnis des Heimatbegriffs, das stark durch ein national-republikanisches Heimatbild geprägt ist. Diese Erkenntnisse werden abschließend vor dem Hintergrund bereits existierender Forschungsergebnisse zum Heimatbegriff und möglicher (wahlkampf-)strategischer Erwägungen diskutiert (siehe Kapitel 6).

\section{2. „Heimat" - Forschungsgegenstand und konzep- tioneller Rahmen}

„Heimat" ist nicht nur Gegenstand politischer und öffentlicher Diskurse, sondern auch eines interdisziplinären Forschungsfeldes, an dem sich diverse sozial- und geisteswissenschaftliche Disziplinen beteiligen (Kühne 20II; Kühne/Schönwald 20I5; Merkel 20I6; Gensheimer/Hieke 20I8). In Österreich erfolgt die Auseinandersetzung 
mit dem Heimatbegriff insbesondere durch die Analyse von "Heimat" in Kunst und Kultur, durch Literatur- und Filmwissenschaften (Huemer 2006; Steiner 1987) sowie hinsichtlich der Einwanderung nach und Auswanderung aus Österreich (Adunka 2002; Orłowski 1993; Wimmer I993). Der zeitliche Fokus liegt meist auf den Zwischenund Nachkriegsjahren, und über alle Disziplinen hinweg finden sich häufig essayistische Abhandlungen zum Begriff (Sebald I99I; Gördesli 20II). Zudem taucht „Heimat" häufig nur im Titel der Arbeiten auf, dient als thematischer Aufhänger oder fungiert als Synonym für Österreich. Strukturierte, explizite Auseinandersetzungen mit dem Begriff selbst und seiner Verwendung in politischen Kontexten erfolgen bisher kaum (Thaler 2013).

In der Forschungslandschaft finden sich unterschiedliche Verständnisse des Heimatbegriffs, die auch in politischen und öffentlichen Diskursen verwendet werden. Ein Ende des Spektrums bildet ein positivistisch-essentialistisches Begriffsverständnis, das sich durch die „unhinterfragte und kritiklose Affirmation des Heimatlichen als Wert an sich" (Kühne/Schönwald 2015) auszeichnet. „Heimat" gilt als exklusiver, stabiler und nicht-wandelbarer Raum und Identifikationsmoment. Am anderen Ende des Spektrums findet sich ein konstruktivistisches Begriffsverständnis, das sich durch die „Ablehnung [von Heimat und, ATH] regionaler Identität als Mittel zur Ausgrenzung Fremder" (Kühne/Schönwald 2015) charakterisieren lässt. „Heimat" wird als integratives, wandelbares und aneigenbares Konzept und als flexibler Identitätsmarker begriffen.

Der vorliegende Artikel versteht den Begriff „Heimat" in einem konstruktivistischen, nicht-essentialistischen Sinn als sozial hergestellt und historisch kontingent. Er legt daher keine Definition von „Heimat“ zugrunde, sondern nähert sich dem Begriff über drei zentrale interdependente Analysedimensionen (Kühne/ Spellerberg 20IO) an, die grundlegende Erkenntnisse der Forschung reflektieren.

(I) „Heimat" bezieht sich häufig sowohl in positivintegrativer als auch in negativ-abgrenzender Hinsicht auf Räume. Diese räumlichen Bezüge erfolgen auf verschiedene Skalen: Der Heimatbegriff tauchte lange meist in lokalen und/oder regionalen Verwendungskontexten auf, oftmals gedeutet als ländliches Idyll (Kühne/ Spellerberg 20IO). Im Nationalsozialismus wurde der Begriff auf die nationale Ebene übertragen (Kühne/ Spellerberg 20IO). In Abgrenzung zu den fortschreitenden Globalisierungsprozessen beobachten ForscherInnen eine Rückbesinnung auf "Heimat" als lokales bzw. regionales Konstrukt (Kühne/Spellerberg 20IO).

(2) Weiters weist der Begriff häufig zeitliche Bezüge auf. Mit "Heimat" kann eine Hinwendung zu und/oder Abgrenzung von einer bestimmten Zeit als Idealzustand oder Sehnsuchtsort verknüpft sein (Kühne 20II; Kühne/ Spellerberg 20IO). So kann „Heimat" einerseits bedrohte oder gar verlorene Situationen oder traditionelle gesellschaftliche Verhältnisse beschreiben. Andererseits kann sich der Begriff auch auf Situationen beziehen, die in der Zukunft liegen und aktiv imaginiert und hergestellt werden sollen (Kühne/Spellerberg 20IO; Kühne 2OII).

(3) "Heimat" erscheint zudem als individuelles, emotionales Konstrukt und abstraktes soziales Phänomen zugleich. Der Begriff integriert Menschen in Gemeinschaften und dient der individuellen und kollektiven Identitätsbildung. „Heimat" konstituiert somit Sozialräume, die ein Gefühl der Geborgenheit entstehen lassen können und emotional gefärbte, starke Identifikationen mit bestimmten Orten oder Regionen sowie Zeiten auslösen (Kühne/Schönwald 20I5). Diese Herausbildung von Identitäten ist in der Regel mit Inklusions- und Exklusionsmechanismen verbunden, die immer wieder mit einer Tendenz zur Höherschätzung des Autochthonen gegenüber dem Allochthonen einhergehen (Kühne/ Schönwald 2015; Kühne/Spellerberg 2010; Kühne 20II).

\section{Diskurstheoretische Konzeptualisierung}

„Heimat" dient somit als Referenzpunkt für individuelle und kollektive Identitäten, die die Grundlage für „vorgestellte Gemeinschaften " (Anderson 2006) bilden. Die der Gemeinschaftsbildung zugrunde liegenden Inklusionsund Exklusionsmechanismen sind mit einer ungleichen Verteilung von Macht verbunden. Daher bietet sich die Konzeptualisierung der Analyse durch die Diskurstheorie von Laclau und Mouffe (200I) an.

Laclau und Mouffe (200I) beziehen sich insbesondere auf die poststrukturalistischen Annahmen von Barthes (1988) und Derrida (1983; 1999) und verstehen Bedeutung als Effekt von Differenzordnungen. Sie entwickeln diese Annahme weiter, indem sie Anleihen bei Foucaults (197I; 1973) Diskursbegriff nehmen und Bedeutungen zudem als fragil, kontingent und kontextvariabel konzeptualisieren (Laclau/Mouffe 200I; Glasze 2015). Diskurse, die Bedeutungen hervorbringen, sind historisch kontingente, einmalige Sagbarkeitsräume und Aussagensysteme, die spezifischen Regeln folgen (Foucault 197I; 1973; Glasze 2008). Ein Diskurs ist - in Anlehnung an Gramscis Hegemoniebegriff - für die Autoren dann besonders erfolgreich, wenn er bestimmte Bedeutungen und damit verknüpfte Weltsichten als natürlich gegeben erscheinen lässt (Laclau/Mouffe 200I). Laclau und Mouffes (200I) Gesellschaftstheorie setzt keine vor-diskursive Ordnung voraus. Alle sozialen Beziehungen werden als temporäre Ergebnisse diskursiver Auseinandersetzungen begriffen.

Auf diesen Überlegungen fußt Laclau und Mouffes (200I) nicht-essentialistische Konzeptualisierung von Identitäten und sozialen Gruppen. Identitäten dienen als Basis für gesellschaftliche Beziehungen und somit soziale Gruppen. Analog zu Diskursen und Bedeutungen 
sind auch Identitäten und soziale Gruppen lediglich partiell und temporär fixiert. Identitäten und soziale Gruppen sind das Ergebnis "sedimentierter Diskurse“ (Laclau 1990), sie sind folglich historische Ergebnisse von politischen Prozessen und basieren nicht auf objektiv gegebenen Tatsachen oder Fakten (Laclau I990; Glasze 2008). In sozialen Gruppen wird eine Grenze gegenüber dem $\mathrm{Au}$ ßen definiert (Laclau/Mouffe 200I; Glasze 2008).

Zentral für die Konstitution sozialer Gruppen auf Basis gemeinsamer Identitäten sind spezifische Knotenpunkte, die so genannten „Signifikanten“ (Laclau I990). Grundlegend für das Verständnis dieses Konzepts ist die aus der strukturalistischen Linguistik stammende Unterscheidung zwischen "Signifikant" (das Bezeichnende bzw. die Buchstabenfolge) und "Signifikat" (das Bezeichnete bzw. das Konzept) (Saussure 200I; Laclau/ Mouffe 200I). Laclau (1990) bezeichnet Signifikanten als "flottierend", da sie nicht fest mit einem Signifikat verbunden sind. Darüber hinaus tragen sie keine spezifische Bedeutung in sich (Laclau 1990). Wenn ihr „Bedeutungshorizont so weit gedehnt ist, dass sie eine breite Zustimmung erfahren" (Dzudzek 20I6), handelt es sich bei Begriffen oder Konzepten um „leere Signifikanten“ (Laclau 1990). Als Knotenpunkte ermöglichen Signifikanten „die Logik der Gleichheit zwischen den Mitgliedern der Gruppe“ (Glasze 2008, 193). „Heimat" wird im vorliegenden Artikel als solcher „leerer Signifikant", der als Basis von Identitäten und sozialen Gruppen fungiert, konzeptualisiert.

\section{Forschungsdesign und Methode}

Auf Basis dieser diskurstheoretischen Überlegungen rekonstruiert der Artikel mittels eines qualitativ-interpretativen Zugangs (Yanow 1996) illustrativ die Heimatverständnisse der beiden Stichwahlkandidaten - Alexander Van der Bellen und Norbert Hofer - im österreichischen Präsidentschaftswahlkampf 2016. Den Ausgangspunkt der Analyse bildete eine Literaturstudie, die mit einer Schlagwortsuche („Heimat“, „home“) in den Literaturdatenbanken Scopus und ISI Web of Science begann und sukzessive durch das Hinzufügen weiterer Schlagworte (bspw. „Deutschland“, „Österreich“) verfeinert wurde. Die Literaturbasis wurde mittels Schneeballtechnik im Forschungsprozess kontinuierlich erweitert.

Wahlkämpfe werden im Artikel als zentrale öffentliche Diskursräume begriffen, in denen sich unterschiedliche „Sprecherpositionen“ (Laclau/Mouffe 200I) artikulieren. Eine multimodale Diskursanalyse, die sprachliche und nicht-sprachliche Verfahren kombiniert, diente der Operationalisierung der diskurstheoretischen Überlegungen (Meier 2008; Glasze 2014). Der sprachliche Teil untersuchte Interviews und TV-Duelle im Wahlkampf (II-I5) sowie Wahlwerbevideos (VI, V2). Da sich Bilder als visuelle Konstruktionen von Wirklichkeit (Renggli 20I4) für die Ergänzung der textlichen Analyse von „leeren Signifikanten“(Laclau I990) gut eignen, wurden für den visuellen Teil zusätzlich die Wahlkampfplakate der Kandidaten, auf denen der Begriff "Heimat" verwendet wurde, einbezogen. Hierzu wurden das Plakat „Aufstehen für Österreich - DEINE HEIMAT braucht DICH JETZT“ aus der Kampagne von Norbert Hofer (PI) sowie die Plakate „HEIMAT BRAUCHT ZUSAMMENHALT“ ( $\left.\mathrm{P}_{2}\right)$ und "WER UNSERE HEIMAT LIEBT, SPALTET SIE NICHT.“ (P3) aus Alexander Van der Bellens Kampagne herangezogen. Im sprachbezogenen Teil der Diskursanalyse wurden die Aussagen der Kandidaten zu „Heimat" in Interviews, TV-Duellen und Videos transkribiert und anschließend durch codierende Verfahren mit Hilfe der Software MAXQDA $^{\oplus}$ ausgewertet. Als deduktive Codes fungierten die aus der Literatur abgeleiteten analytischen Dimensionen räumliche Bezüge, zeitliche Bezüge und Identitäten (siehe Kapitel 2). Die Stellen des Materials, die zu den deduktiven Codes passten, wurden diesen beim Lesen zugeordnet. Anschließend wurden die entsprechenden Textstellen zusammengestellt, nochmals gelesen und im Zuge dessen mit induktiven Sub-Codes versehen, um die Codierung zu verfeinern. Zusätzlich wurden beim Lesen der Transkripte induktive Codes vergeben. Die induktiven Codes bezogen sich insbesondere auf rhetorische Strategien der beiden Stichwahlkandidaten: Es wurden thematische Bezüge, normative Bezüge sowie emotionale und symbolische Bezüge als Codes festgelegt. Auch diese Textstellen wurden gesammelt ausgegeben und beim nochmaligen Lesen mit spezifischen induktiven Sub-Codes versehen. Im Anschluss erfolgte eine weitere Codierungsrunde des gesamten Materials mit allen deduktiven und induktiven Codes sowie ihren jeweiligen SubCodes (siehe Tabelle I).

Als räumliche Bezüge wurden jene Textstellen codiert, die den Begriff "Heimat“ zu einer geographischen Einheit und/oder Skala in Bezug setzten, indem sie ihn beispielsweise auf die europäische, nationale oder regionale Ebene bezogen. Der Code zeitliche Bezüge wurde Textstellen zugewiesen, die den Heimatbegriff rhetorisch mit Vergangenheit, Gegenwart oder Zukunft, beispielsweise als gegebenen Idealzustand oder aneigenbare Wunschvorstellung, verknüpften. Als thematische Bezüge wurden all jene Textstellen codiert, in denen die Stichwahlkandidaten den Begriff „Heimat" mit konkreten politischen Themen, gesellschaftlichen Herausforderungen und/ oder spezifischen Politikfeldern, wie Sicherheits-, Arbeitsmarkt- oder Bildungsfragen, in Zusammenhang brachten. Normative Bezüge wurde als Code verwendet, wenn sich die Stichwahlkandidaten bei der Beschreibung von "Heimat" auf individuelle und kollektive Werte und Normen, wie Offenheit oder Gleichheit, bezogen oder bestimmte Merkmale als positive oder negative Werte thematisierten. Darüber hinaus zeigten sich im 
Tabelle 1: Zusammenstellung der deduktiven und induktiven Codes sowie exemplarischer Sub-Codes

\begin{tabular}{ll}
\hline Codes & Sub-Codes (exemplarisch) \\
\hline Räumliche Bezüge & $\begin{array}{l}\text { Internationale Ebene; europäische Ebene; nationale Ebene; Bundesland; lokal; Stadt; Land; andere } \\
\text { räumliche Bezugspunkte } \\
\text { Zeitliche Bezüge }\end{array}$ \\
$\begin{array}{l}\text { Vergangenheit; Gegenwart; Zukunft; aneigenbar; gegeben; Idealvorstellung } \\
\text { Thematische Bezüge }\end{array}$ & $\begin{array}{l}\text { Arbeit \& Beschäftigung; Wohlstand; Sicherheit \& Neutralität; direkte Demokratie; Bildung; Migration \& } \\
\text { Integration }\end{array}$ \\
Normative Bezüge & Offenheit; Veränderung; Bewahren; Gleichheit; Aktivität; Zusammenhalt; Kooperation \\
Emotionale und & Liebe; Geborgenheit; Stolz; Ruhe; Aufgehobenheit; Alpen; Nationalflagge; Dialekt; Trachten; \\
symbolische Bezüge & Muttersprache; Vaterland \\
Identitäten & Individuelle Identitäten; kollektive Identitäten; Inklusivität; Exklusivität \\
\hline
\end{tabular}

Textmaterial emotionale und symbolische Bezüge, wenn die Kandidaten eigene oder fremde Emotionen auf positive oder negative Weise thematisierten, um ein spezifisches Bild und Verständnis von „Heimat“ zu vermitteln. Hierzu zogen die Stichwahlkandidaten beispielsweise Liebe oder Geborgenheit heran oder nahmen auf die alpenländische Bergwelt und die österreichische Nationalflagge Bezug. Der Code Identitäten wurde vergeben, wenn in den Aussagen zum Heimatbegriff implizit oder explizit spezifische individuelle und/oder kollektive Identitätsmarker auftauchten.

Die deduktiven und induktiven Codes räumliche $\mathrm{Be}$ züge, zeitliche Bezüge, thematische Bezüge, normative Bezüge sowie emotionale und symbolische Bezüge fungieren gleichzeitig als Analysedimensionen. Bei der Unterscheidung zwischen den Dimensionen handelt es sich um eine rein analytische Trennung. Sie werden untereinander nicht als hierarchisch, sondern als netzwerkartig ko-konstitutiv begriffen. Durch den gleichzeitigen Einsatz unterschiedlicher Bezüge können sich diese wechselseitig untermauern und verstärken. Im komplexen Wechselspiel tragen die fünf Dimensionen dazu bei, bestimmte Identitäten bei den RezipientInnen zu konstituieren und/ oder bereits vorhandene Identitäten anzusprechen und zu verstärken.

Für die ergänzende visuelle Komponente der Diskursanalyse wurde auf Methoden der Politischen Ikonographie zurückgegriffen (Bernhardt/Liebhart 20I7). Die Wahlplakate, auf denen der Heimatbegriff auftaucht, wurden ebenfalls in MAXQDA $^{\circledR}$ geladen und für spezifische Plakatausschnitte ebenfalls Codes und Sub-Codes vergeben. So wurden beispielsweise Aufbau, räumliche Anordnung, Perspektiven, Farben, Thema, Inhalt, Narrationen und Besonderheiten der jeweiligen Plakate codiert. Diese Codes wurden anschließend den deduktiven und induktiven Codes sowie ihren jeweiligen Sub-Codes, die für das Textmaterial vergeben wurden, zugeordnet. Im Fall des Wahlplakates PI von Norbert Hofer wurde beispielsweise der Hintergrund mit räumliche Anordnung/ im Hintergrund und Inhalt/Nationalflagge codiert und anschließend außerdem den Codes räumliche Bezüge/national sowie symbolische Bezüge/Nationalflagge aus dem Textmaterial zugeordnet.

Abschließend wurden die mit deduktiven und induktiven Codes markierten Textstellen und Plakatausschnitte der jeweiligen Kandidaten nochmals durch das Programm ausgegeben und durch die wiederholte Betrachtung die Aussagen und Bildsprache zum Heimatbegriff der Kandidaten interpretiert und verglichen, um Ähnlichkeiten und Unterschiede zwischen ihren Begriffsverständnissen zu identifizieren.

\section{Eine Heimat oder mehrere Heimaten?}

Die Analyse zeigt, dass die Stichwahlkandidaten Alexander Van der Bellen und Norbert Hofer verschiedene Verständnisse von „Heimat“ repräsentieren. Diese Unterschiede werden entlang der interdependenten Dimensionen räumliche Bezüge (siehe Kapitel 5.I), zeitliche Bezüge (siehe Kapitel 5.2), thematische Bezüge (siehe Kapitel 5.3), normative Bezüge (siehe Kapitel 5.4), emotionale und symbolische Bezüge (siehe Kapitel 5.5) illustriert. Verbindungen zwischen diesen werden exemplarisch beleuchtet. Anschließend wird dargelegt, welche spezifischen Identitäten (siehe Kapitel 5.6) durch die Referenzen konstituiert bzw. verstärkt werden (sollen).

\subsection{Räumliche Bezüge}

Beide Kandidaten beziehen sich in ihren Ausführungen im Vergleich zu den anderen Analysedimensionen relativ häufig auf spezifische Räume. Van der Bellens räumliche Bezüge sind mehrdimensional. Als Referenzpunkte für sein Heimatverständnis fungieren unterschiedliche Skalen, von der lokalen Ebene über die Bundesländerebene bis hin zur transnationalen Ebene (I4, I5). Er bezieht sich beispielsweise gleichwertig auf 
die lokale bzw. regionale sowie die Bundesländerebene, wenn er auf die Frage „Was verbinden Sie - welchen Ort konkret - verbinden Sie mit Heimat?" (I5) antwortet: „Kaunertal, Tirol, Innsbruck, Linz, Wien“ (I5). Im Kontext des Heimatbegriffs nimmt Van der Bellen außerdem auf die Europäische Union und Europa Bezug (V2, I4). Multiskalare Referenzen finden sich bei Van der Bellen auch auf der lokalen Ebene hinsichtlich ländlicher und städtischer Räume. „Heimat" ist für ihn nicht auf ländliche Räume beschränkt, da sie sowohl in ländlichen als auch in städtischen Gebieten zu finden ist (I3).

Trotz multipler Raumbezüge zeigt sich eine zentrale Rolle des Kaunertals, des Bundeslandes Tirol und ländlicher Räume. Eine Interviewaussage des Kandidaten belegt exemplarisch, dass das Bundesland Tirol gegenüber der nationalen Referenzebene als „Heimat" für Van der Bellen wichtiger ist: „Ich sage, Österreich - zuerst Tirol, dann ganz Österreich - hat mir eine Heimat gegeben, hat mir eine Heimat geschenkt, wenn man so will“ (I5). Derartige räumliche Bezüge werden durch symbolische Bezüge (hierzu allgemein siehe Kapitel 5.5) mittels der Hintergrundgestaltung der Wahlplakate als alpenländische Bergkulisse $\left(\mathrm{P}_{2}, \mathrm{P}_{3}\right)$ illustriert und verstärkt.

Eine besonders zentrale Rolle nimmt in den raumbezogenen Ausführungen Van der Bellens das Kaunertal ein (II, I3, I4, I5, V2). Es hat für ihn eine besondere biographische Bedeutung, da er dort aufgewachsen ist. Das Kaunertal und die Funktionslogiken in den dortigen Dorfgemeinschaften dienen wiederholt als positive Beispiele oder sogar als Analogie für ganz Österreich (I4, I5). Obwohl Van der Bellen städtische und ländliche Räume als Heimaträume begreift, haben ländliche Räume und insbesondere die Alpenregion für ihn eine spezifische Bedeutung: „Ich bin begeisterter Großstädter. Aber ich komme vom Dorf" (I3).

Hofer beantwortet die Frage nach der räumlichen Verortung seiner "Heimat" im Vergleich zu Van der Bellen durch eine eindimensionale Antwort. Er nimmt ausschließlich auf die nationale Ebene Bezug, nicht auf mehrere Ebenen, Orte oder Regionen (II, I3, I5, VI). Dies wird beispielsweise in einer Aussage Hofers in einem TV-Duell deutlich: „Tarek Leitner: ,Wo ist Ihr Ort der Heimat, Herr Hofer?' Norbert Hofer: ,In Österreich. Das ist meine Heimat $[\ldots]^{\text {“. }}(\mathrm{I} 5)$.

Hofers Wahlplakat, das den Begriff „Heimat“ aufgreift, bildet diese Fokussierung auf die nationale Ebene ebenfalls ab, da die österreichische Nationalflagge als Hintergrund des Plakats dient (PI). Auch bei Hofer zeigt sich hier, wie symbolische Bezüge die räumlichen Bezüge unterstützen und verstärken können. Eine nähere Beschreibung dieser räumlichen Dimension seiner „Heimat Österreich" bleibt in den analysierten Transkripten weitestgehend aus (II, I2, I3, I5, PI).

Die lokale Ebene spielt als räumliche Referenz eine sehr untergeordnete Rolle. Nur selten spricht Hofer bei- spielsweise - in einem anderen inhaltlichen Kontext - über seine „Heimatgemeinde“ (I2) Pinkafeld im Burgenland. In seinen Ausführungen zu „Heimat“ lässt sich keine explizite Unterscheidung oder eine Gleichsetzung von städtischen und ländlichen Räumen als "Heimat" erkennen (I2, I3, I5).

\subsection{Zeitliche Bezüge}

Wenn die Stichwahlkandidaten über „Heimat" sprechen, beziehen sie sich sowohl auf die Vergangenheit als auch auf die Zukunft. Sie leiten aus den zeitlichen Bezügen konkrete Aktivitäten und verantwortliche Akteure ab. Die Vergangenheit ist in Van der Bellens Aussagen meist positiv konnotiert. Er bezieht sich überwiegend auf seine Kindheit und Jugend im Kaunertal (I3, I4, I5, V2). Allerdings spricht er auch vereinzelt negativ über die Vergangenheit, wenn er sich auf die bisherige Verwendung des Begriffs „Heimat" durch rechte Parteien oder rechtsextreme Gruppierungen bezieht (I4, I5). So äußert er sich in einem Interview kritisch zur Instrumentalisierung des Heimatbegriffs durch die Nationalsozialisten: „Es ist sicher richtig, dass die Nazis mit ihrer Deutschtümelei dazu beigetragen haben, den Heimatbegriff zu diskreditieren" (I4).

Für die Zukunft seiner „Heimat" zeichnet Van der Bellen ein positives Bild. Er begreift sie als aktiv gestaltbar, aneigenbar und fortschrittlich (I3, I4, I5, V2). Um Österreich in eine positive Zukunft zu führen, sind alle dort lebenden Personen gefragt. Die Verantwortung wird nicht spezifischen Personengruppen zugeschrieben $\left(\mathrm{I}_{3}, \mathrm{I}_{4}, \mathrm{I}_{5}, \mathrm{~V} 2\right)$.

Auch in Hofers Aussagen ist die Vergangenheit positiv besetzt. Ohne konkrete Beispiele anzuführen bezieht sich Hofer auf die Vergangenheit als etwas zu Bewahrendes (I5, VI). Er betont einen Dualismus aus der positiven Vergangenheit Österreichs und den nötigen Verbesserungen in der Zukunft. Dies verdeutlicht eine Aussage aus seinem Wahlkampfvideo: „Man muss einerseits die guten Dinge bewahren... Viele sagen: ,Wir wollen unser Österreich zurück'. Und andererseits auch Österreich in eine gute Zukunft führen“(VI).

Hofer vermittelt ebenfalls das Bild einer positiven und gestaltbaren Zukunft. Verbesserungen in Österreich beschreibt er in seinem Wahlkampfvideo als zentrale Motivation für seine Kandidatur, bleibt hierbei jedoch eher abstrakt: „Wenn Sie sagen, was treibt dich an, dann ist es einfach so, dass man sieht, was in diesem Land besser sein kann" $(\mathrm{VI})$. Auch der in die Ferne und symbolisch in die Zukunft gerichtete Blick des Kandidaten auf seinem Wahlplakat unterstreicht - in national-republikanischer Tradition - diese Zukunftsorientierung (PI). Entsprechend präsentiert er sich selbst - in seiner möglichen zukünftigen Rolle als Bundespräsident - als einen zentralen Verantwortungsträger für diese Zukunft: 
„Mein Beitrag wird vielleicht sein, Bundespräsident zu sein“ (VI). Darüber hinaus spricht Hofer ebenfalls einen breiten Kreis an VerantwortungsträgerInnen an, indem er diejenigen, die in Österreich leben und ein gutes Leben führen wollen, als Verantwortungsträger benennt (VI, II).

\subsection{Thematische Bezüge}

Die Kandidaten verknüpfen den Begriff „Heimat“ mit unterschiedlichen Themen. Außerdem machen beide den Begriff "Heimat" zum Thema, um sich vom jeweils anderen Kandidaten abzugrenzen. Van der Bellen adressiert besonders die Bereiche Migration und Integration (II, I3, I4, I5, VI). Migration ist seiner Ansicht nach positiv, wünschenswert und normal. Integration kann gelingen. Um dies zu illustrieren rekurriert er, unter anderem, auf seine persönlichen Fluchterfahrungen: „Vor kurzem stellte der Kaunertaler Bürgermeister einen Film über die Entwicklung des Tals vor und sagte in seiner Ansprache auch ein paar Worte zu mir: ,Der isch als Flüchtlingskind kemma, und iatz isch er oana von uns.“" (I4). Seine Aussagen sind eher allgemein gehalten und verzichten auf konkrete Lösungsvorschläge (I3, I4).

Van der Bellens Ausführungen zu Migration und Integration beschränken sich nicht auf biographische Bezüge. Er spricht auch die Migration anderer Menschen, vor allem angesichts der Fluchtbewegungen nach $\mathrm{Eu}-$ ropa im Jahr 2015, an und unterstützt seine Argumente durch zeitliche Referenzen (I3, I5). So beschreibt er die historische Dimension Österreichs als Einwanderungsland und Vielvölkerstaat in einem TV-Duell: „Österreich $[\ldots]$ hat mir eine Heimat gegeben [...]. Anderen wurde eine Heimat genommen. Zum Beispiel manchem Flüchtling, der jetzt versucht, nach Europa zu kommen. [...] Da hat, finde ich, Österreich [...] eine ganz hohe Integrationskraft bewiesen. Nicht seit 20 Jahren, seit 150 Jahren. Mindestens. Wenn ich bedenke, wer alles zugezogen und wieder weggezogen ist. Die Tschechen und Slowaken, die Bosnier und aus Galizien seinerzeit [...]" (I5). Neben Migration und Integration spricht Van der Bellen Jugendliche als zukünftige Generationen an, für die die Heimat lebenswert sein soll (V2). Er benennt Arbeit, Wohlstand und Frieden als erstrebenswerte Ziele für ein zukünftiges Österreich (V2).

Außerdem thematisiert Van der Bellen den Begriff "Heimat" und seine historische Verwendung (siehe auch Kapitel 5.2). Sein inhaltliches Anliegen der Zurückweisung der Vereinnahmung des Heimatbegriffs als etwas nicht mehr Zeitgemäßes unterstützt er durch zeitliche Referenzen (I5). Er betont besonders, dass die bisherige Vereinnahmung des Begriffs durch konservative und rechte Parteien und Gruppierungen in einem zu engen Begriffsverständnis resultierten: „[D]ieser Begriff ist nicht ideologisch besetzt [...]. Das haben wir uns nur alle einreden lassen von der FPÖ. Aber ich hab das nie eingesehen, warum man das Monopol auf Heimat deutschtümelnden Burschenschaften ausgerechnet überlassen soll“ (I5). Van der Bellen lehnt ein solch enges Begriffsverständnis und die Monopolisierung des Begriffs ab (I4, I5). Außerdem betont er sein offenes Begriffsverständnis, beispielsweise im Rahmen eines Interviews: „Ja, man öffnet den Blick nach außen. Das finde ich gut. Ich habe immer gesagt, dass mein Heimatbegriff nicht ausschließend, sondern einschließend ist" (I4).

Im Vergleich zu Van der Bellen verknüpft Hofer ein noch breiteres Themenspektrum mit dem Begriff „Heimat". Hierzu zählen die Bereiche Sicherheit, Migration, Regierungsbildung, Bildung, Freihandel, direkte Demokratie, Jugend, Arbeit und Drogen (II, I2, I3, I5, VI). Auch er beschränkt sich zumeist auf grundsätzliche Zustimmung, Ablehnung, Perspektiven und Problemdiagnosen.

Innere und äußere Sicherheit spielen in Hofers Ausführungen eine besonders zentrale Rolle (I3, I5, VI). Beispielsweise signalisiert er gleich zu Beginn seines Wahlkampf-Videos die große Bedeutung von Sicherheit für seine „Heimat" Österreich: „Was ist das Wichtigste in Österreich? [...] Und ich glaube das ist Sicherheit" (VI). In einem TV-Duell thematisiert er auch die Rolle des Bundesheers zur Wahrung der, traditionellen' Neutralität, in dem er betont, dass er, im Gegensatz zu seinem Gegenkandidaten, das Bundesheer unter keinen Umständen den Vereinten Nationen unterstellen würde, um die Sicherheit Österreichs zu gewährleisten (I5).

Auch für Hofer spielen die Themen Migration und Integration eine wichtige Rolle im Zusammenhang mit „Heimat". Migration und Integration werden als nur begrenzt möglich, negativ und folgenreich dargestellt. Dies verdeutlicht eine Interviewaussage des Kandidaten: „Sie sagen diese Integrationsfähigkeit, die es in Österreich gibt... Ja, die gibt es, die gibt es tatsächlich, nur sind wir massiv überfordert. [...] Wir haben in vielen Bereichen in der zweiten Generation - also die, die eigentlich schon länger da sind - größere Integrationsschwierigkeiten $[\ldots]^{\prime \prime}(\mathrm{I} 5)$.

Über alle Themen hinweg spricht Hofer häufig entweder ausschließlich oder insbesondere ÖsterreicherInnen an. Dies zeigt sich am Beispiel Arbeit: „Ich habe miterlebt, wie hart die Österreicherinnen und Österreicher arbeiten - Nachtdienst, Schichtdienst, viele Überstunden oft, oft mit einer sehr geringen Bezahlung [...] das muss man sehen" (VI). Auch hier zeigt sich die wechselseitige Verstärkung unterschiedlicher Referenzen, indem Hofer sein inhaltliches Anliegen mit Emotionen verknüpft (zu emotionalen Bezügen siehe Kapitel 5.5).

Darüber hinaus versucht Hofer in den Duellen durch die Verknüpfung verschiedener Themen eine deutliche Abgrenzung von seinem Gegenkandidaten zu erreichen. Hierfür verweist er auf zu seinen eigenen politischen Po- 
sitionen konträren Standpunkte, insbesondere der Grünen Partei (I5), und macht auch das Heimatverständnis seines Gegenkandidaten zum Thema. So vermittelt er in einer Interviewpassage, die sich um den Migrationshintergrund Van der Bellens und das Verhältnis der Grünen zum Heimatbegriff dreht, seine Ablehnung der Cannabis-Legalisierung: „Ja schauen Sie [Herr Van der Bellen, ATH], natürlich ist das... Ich hoffe, dass Österreich Ihre Heimat ist. Aber, dass gerade Sie diesen Begriff plakatieren... [...] Das ist so als würde ich plakatieren ,Cannabisfreigabe für alle؛. Das wäre genauso unglaubwürdig. Ich halte das [die Verwendung des Heimatbegriffs durch Van der Bellen, ATH], also gelinde gesagt, für eigenartig“ (I5).

\subsection{Normative Bezüge}

Die Werte, die die beiden Stichwahlkandidaten im $\mathrm{Zu}$ sammenhang mit "Heimat" vermitteln, sind ähnlich, erhalten jedoch durch ihre Kontextualisierung unterschiedliche Bedeutungen. Van der Bellen betont insbesondere die Werte Gleichheit und Gleichwertigkeit, Zusammenhalt und Kooperation, Aktivität und Veränderung. Diese Werte sind bei ihm positiv konnotiert und unterstreichen die soziale Dimension von "Heimat" (II, I3, I4, I5, VI, P2, P3). Zentral für ein gutes Leben und Zusammenleben in der "Heimat" seien die Gleichheit und Gleichwertigkeit aller Menschen, die dort leben. Dies verdeutlicht beispielsweise eine Aussage, die er in einem Wahlkampf-Video zu „Heimat" trifft: „Hier im Kaunertal hab' ich gelernt, dass jeder Mensch gleich viel Wert ist" (V2). Kein Mensch und keine Personengruppe soll seiner Ansicht nach von der Gemeinschaft ausgeschlossen werden (II, I5, V2). Gleichheit und Gleichwertigkeit bezieht Van der Bellen sowohl auf ÖsterreicherInnen als auch auf Nicht-ÖsterreicherInnen, die im Land leben bzw. leben möchten (II, I5).

Kooperation und Zusammenhalt stellen für ihn weitere zentrale Bausteine einer funktionierenden heimatlichen Gemeinschaft dar. Dies verdeutlicht Van der Bellen in seinem Wahlkampf-Video: „Soll keiner meinen, dass er keinen anderen braucht. Wir sind aufeinander angewiesen. Wir brauchen einander" (V2). Er wendet sich explizit gegen eine Spaltung der österreichischen Gesellschaft (II, I3, P3, V2). Die Slogans der Wahlkampfplakate unterstreichen mit "Heimat braucht Zusammenhalt" ( $\left.\mathrm{P}_{2}\right)$ und "Wer unsere Heimat liebt, spaltet sie nicht" $\left(\mathrm{P}_{3}\right)$ diese Position.

Um die heimatliche Gemeinschaft aufrechtzuerhalten, ist für Van der Bellen die wechselseitige Aktivität sowohl der Aufgenommenen als auch der Aufnehmenden nötig (II, I3, I4, V2). Dies wird beispielsweise in einem Interviewzitat deutlich: „Aber Heimat kann man sich nicht nehmen, sie muss einem auch gegeben werden. Ich könnte das Kaunertal nicht als meine Heimat bezeichnen, wenn die Menschen dort mich nicht so freundlich aufgenommen hätten. [...] Das ist ja ein beidseitiger Prozess" (I4). Auch dieses Beispiel führt vor Augen, wie Van der Bellen Werte und Normen mit räumlichen Bezügen verknüpft, um sein offenes Verständnis von „Heimat“ zu vermitteln.

Van der Bellens "Heimat" ist von Veränderung geprägt. Er bewertet sie als grundsätzlich positiv, aber auch als herausfordernd. Die Offenheit für Neues stellt in Van der Bellens Augen eine Grundvoraussetzung für positive Veränderungen dar (II, I4). In Einklang mit dieser Position weist der Kandidat auch die Beharrung auf Althergebrachtem zurück. Die positive Einstellung gegenüber einer sich verändernden „Heimat" verdeutlicht eine Interviewpassage zur Migration nach Wien: „Der Zuzug ging sehr schnell, das erschwert die Anpassung. [...] Aber die Welt verändert sich, und es hilft leider nicht, darauf zu beharren, dass alles so bleiben muss, wie es war" (I4).

Hofer adressiert in seinen Aussagen zum Thema „Heimat" ebenfalls die Werte Gleichheit und Gleichwertigkeit sowie Aktivität, bezieht durch eine andere Kontextualisierung jedoch andere Standpunkte. Außerdem thematisiert auch er Veränderung, jedoch im Zusammenspiel mit einer stark bewahrenden Komponente.

Hofer adressiert das Thema Gleichheit, indem er betont, dass „[...] jeder, der gern hier lebt, [...] einen Beitrag leisten“ (VI, II) muss. Er verknüpft den Heimatbegriff auf diese Weise mit dem Leistungsprinzip. Immer wieder richtet sich der Kandidat an die "Österreicherinnen und Österreicher" (I2, I4, I5, VI) und hebt deren Leistungen in den Interviewaussagen und im Wahlkampfvideo hervor. Durch die Bezugnahme auf die Prinzipien Leistung und Verantwortung werden auch die Werte Aktivität und Eigeninitiative betont (VI, II). Dies geschieht jedoch auf andere Weise als bei seinem Gegenkandidaten Van der Bellen, der einen eher kooperativen Aktivitätsgedanken vertritt. Aktivität und Engagement werden auch durch das Wahlplakat Hofers zum Heimatbegriff unterstrichen. Es postuliert den Slogan „Aufstehen für Österreich - Deine Heimat braucht dich jetzt" ${ }^{\text {" }}$ II).

Als weitere zentrale Werte in Zusammenhang mit "Heimat" spricht Hofer einen Dualismus aus Bewahren und Veränderung an. Zentral ist für ihn das Bewahren des positiven Status Quo - und teilweise eines früheren Österreichs - und insbesondere dessen, was ÖsterreicherInnen über viele Generationen hinweg geleistet und geschaffen hätten (VI, I4, I5). So konstatiert Hofer in einem Interview: „Man muss einerseits die guten Dinge bewahren. Viele sagen: ,Wir wollen unser Österreich zurück“" (VI). Gleichzeitig solle Österreich positiv weiterentwickelt werden $\left(\mathrm{I}_{5}, \mathrm{VI}\right)$. Veränderung ist jedoch in Hofers Aussagen nicht ausschließlich positiv besetzt. Insbesondere durch den Zuzug von MigrantInnen sind positiven Veränderungen seiner Ansicht nach Grenzen gesetzt. So betont er die begrenzte Integrationsfähigkeit Österreichs: „Und ich bin der Meinung, dass wir bei 
diesen schieren Mengen, die jetzt kommen, alles das verspielen, was unsere Väter, Großväter, Großmütter, Mütter unter großen Entbehrungen erwirtschaftet und aufgebaut haben. [...] Wir können das nicht schaffen, wenn in den nächsten Io Jahren eine Million Menschen nach Österreich zusätzlich kommen sollen" (I5). Zeitliche Referenzen und Emotionen dienen Hofer hier dazu, um sein Bild von "Heimat" und die damit verknüpften Werte noch deutlicher zu vermitteln.

\subsection{Emotionale und symbolische Bezüge}

Beide Stichwahlkandidaten setzen wiederholt Emotionen und Symbole ein, um ihre Botschaften zu vermitteln und/oder zu verstärken. Van der Bellen setzt häufig auf positive Emotionen, wenn er über „Heimat" spricht. In diesem Zusammenhang wählt er oftmals Worte, die ein Gefühl der Geborgenheit erzeugen sollen. So spricht der Stichwahlkandidat vor allem über seine eigene „Heimat“ und verknüpft diese mit den Gefühlen „wohlfühlen“ (II), "akzeptiert werden“ (II) sowie „Ruhe“ (I4) und „Aufgehobenheit" (I4).

Durch emotionale Bezüge verdeutlicht Van der Bellen, dass "Heimat" nicht notwendigerweise auf einen Ort, eine Region oder eine andere geographische Skala bezogen sein muss (II, I4, I5). „Heimat" wird in seinen Augen auch durch Gefühle und die Menschen hergestellt, die an einem bestimmten Ort leben und diese Gefühle hervorrufen. So beschreibt er "Heimat" in einem Interview beispielsweise als Ort, ,[w]o man sich wohlfühlt, wo man aufgenommen wird, wo man angenommen wird" (II). Auf diese Weise unterstreicht er neben der affektiven Komponente von "Heimat" auch nochmals die soziale.

Van der Bellen vermittelt nicht nur positive emotionale Assoziationen, sondern weist auch bestimmte Gefühle gegenüber seiner „Heimat" zurück. So wird er beispielsweise gefragt, ob er auf Österreich "stolz“ (I4) sei. Daraufhin antwortet er: „Mit dem Begriff muss man vorsichtig sein. Was heißt das, stolz zu sein? Beeindruckend ist die Entwicklung seit 1945 auf jeden Fall. [...] Man kann ohne Übertreibung sagen, dass die Menschen in den vergangenen 70 Jahren etwas Tolles geleistet haben" (I4).

Neben Emotionen setzt Van der Bellen auf Symbole, um seine Version von "Heimat", insbesondere als Alpenidylle, zu illustrieren. Besonders häufig rekurriert er hierbei auf den kaunertalerischen Dialekt sowie Berglandschaften (II, I3, I5, V2), die seine räumlichen Bezüge untermauern (siehe auch Kapitel 5.I). Er bezieht sich häufig auf alpenländische Bergkulissen oder auf „die Berge“ (I4). Dieser Eindruck wird durch seine Wahlplakate noch verstärkt. Bei den Plakaten mit direktem Heimatbezug dient ein unscharf dargestelltes alpenländisches Bergpanorama als Hintergrund ( $\left.\mathrm{P}_{2}, \mathrm{P}_{3}\right)$. Auf diese
Weise betont der Kandidat seine Verbindung zu ,klassischen' österreichischen Motiven. Damit geht eine gewisse Romantisierung des ländlichen, alpinen Österreichs als Heimatraum einher.

Oftmals setzt Van der Bellen Emotionen und Symbolik zusammen ein. So stellt er "Heimat" beispielsweise als einen Ort des Wohlfühlens und des Dialektsprechens dar: „Das ist für mich alles Heimat - Heimat ist da, wo ich mich wohlfühle, wo ich die Sprache spreche, den Dialekt vor allem, das ist etwas besonders Heimatliches - in Wien bin ich nicht so perfekt, aber im Kaunertal schon" (I5).

Hofer greift nur vereinzelt auf positiv besetzte Emotionen zurück. Er spricht beispielsweise von Liebe zu Österreich (I5). Wie auch Van der Bellen betont er die Bedeutung von Geborgenheit und Wohlgefühl in der ${ }_{\text {"Heimat" }}$ (I3, I5). Dies wird in einer Interviewaussage im Rahmen eines TV-Duells deutlich: „Das [Österreich, $\mathrm{ATH}]$ ist meine Heimat. Die liebe ich, hier fühle ich mich wohl und hier fühle ich mich geborgen" (I5). Darüber hinaus setzt Hofer auch negative Assoziationen, wie "große Entbehrungen“ (I5), ein. Durch den gleichzeitigen Einsatz solcher positiven und negativen Emotionen versucht er zu unterstreichen, dass Österreich und die Leistungen der ÖsterreicherInnen über Generationen hinweg bewahrt werden sollen (I3, I5).

Als zentrales Symbol hinsichtlich seines Heimatverständnisses erweist sich bei Hofer die österreichische Nationalflagge (PI, VI, I5). Diese taucht sowohl in seinen Interviewaussagen (I3, I5) als auch in seiner Plakatkampagne zum Heimatbegriff als großflächiges Hintergrundmotiv auf (PI). Zusätzlich betont wird diese nationale Symbolik durch den wiederholten Einsatz des Terminus „unser Österreich“ (I3, I5, VI) als Symbol für einen traditionellen österreichischen Wertekanon, ohne diesen jedoch konkret zu benennen. Durch den Einsatz von Emotionen und Symbolen untermauert und verstärkt auch Hofer seine räumlichen Bezüge auf die Nation bzw. die Republik Österreich als zentralen Heimatraum.

Darüber hinaus versucht Hofer die Glaubwürdigkeit seines Stichwahlgegners zu unterminieren, indem er dessen Symbolik hinterfragt. Er stellt beispielsweise das Tragen von Trachten im Wahlkampf und das Sprechen des kaunertaler Dialekts durch Van der Bellen als unglaubwürdig dar (I3, I5). In einem Fernsehduell der beiden Stichwahlkandidaten versucht er beispielsweise zu zeigen, dass sein Gegenkandidat sich die traditionelle österreichische Symbolik lediglich aufgrund wahltaktischer Erwägungen aneignet: „Jeder muss für sich selbst entscheiden, wie er den Begriff ,Heimat' lebt. Nur, man muss halt vergleichen: Was habe ich in diesem Wahlkampf gemacht und was habe ich in den Jahren davor gemacht?' Aber, wenn Van der Bellen für sich entschieden hat, eine Tracht zu kaufen, dann gratuliere ich ihm. Weil, 
eine Tracht ist deswegen gut, weil Sie nicht der Mode unterliegt" (I3). Gleichzeitig betont er, dass er selbst solche expliziten Betonungen österreichischer Brauchtümer und Traditionen nicht nötig habe, da er diese in seinem Alltag und Berufsleben stetig auch außerhalb von Wahlkampf-Phasen - und daher glaubwürdiger - hochhalte $(\mathrm{I} 3, \mathrm{I} 5)$.

\subsection{Identitäten}

Durch das Zusammenspiel der verschiedenen interdependenten Referenzen im Zusammenhang mit dem Heimatbegriff (siehe Kapitel 5.I-5.5) machen die beiden Stichwahlkandidaten individuelle und kollektive Identitätsangebote. Allerdings unterscheiden sich diese hinsichtlich der Charakteristika, der angenommenen Beständigkeit sowie der Möglichkeit multipler Identitäten. Van der Bellens Ausführungen zum Heimatbegriff repräsentieren multiple Identitätsangebote. Seine Identitätsangebote sind insbesondere mit Aussagen zu seiner persönlichen Heimat verknüpft. So stellt sich Van der Bellen als Kaunertaler, als Tiroler, als Wiener, als Österreicher und als Europäer dar (I4, I5). Gleichzeitig hebt er seinen Migrationshintergrund als Geflüchteter hervor (I4). Neben multiplen räumlichen Bezügen betont er als zentrale Pfeiler der Identitäten durch thematische, normative und emotionale Bezüge das Gemeinschaftliche, das auf keine Gruppe beschränkt ist und für alle gilt, die "Heimat" suchen und sich aktiv an ihrer Gestaltung beteiligen möchten (II, I3, I5, V2). Identitäten sind nicht an die Staatsbürgerschaft gebunden. Stattdessen beschreibt Van der Bellen Identitätsbildungen als soziale Prozesse und betont deren Inklusivität und Dynamik. Dies unterstreicht die multiplen Konstitutionsmechanismen und die Offenheit für Neues und zeigt dass er Identitäten als dynamisch betrachtet und, dass eine Person mehrere Identitäten haben kann (I3, I4, I5, V2).

Hofer stellt ein engeres, eindimensionaleres Identifikationsangebot bereit als sein Gegenkandidat, indem er sich ausschließlich als Österreicher präsentiert (I3, I5, PI, VI). Grundsätzlich überwiegt in seinen Aussagen ein kollektives Identifikationsangebot, das die nationale Ebene und die nationale Gemeinschaft besonders betont (I3, I5, PI, VI). Er rekurriert auf die Österreichische Republik und die österreichische Staatsbürgerschaft als zentrale Identifikationsmarker, die insbesondere durch räumliche, symbolische und thematische Referenzen konstituiert und verstärkt werden ( I3, I5, PI, VI). Er stellt österreichische Werte, Leistungen und Integrität in den Vordergrund und porträtiert die ,traditionelle österreichische Identität als stabil und bewahrenswert. Veränderungen dieser Identität seien nur in engen Grenzen wünschenswert (I3, I5). Insgesamt vermittelt Hofer ein statisches, stärker naturalistisches und exklusives Bild von Identitäten.

\section{Diskussion und Conclusio}

Der vorliegende Artikel rekonstruiert die konkurrierenden Verständnisse von "Heimat" im Kontext der Stichwahl um das Amt des Bundespräsidenten 2016. Die Konzeptualisierung von "Heimat" als leerer, flottierender Signifikant hat sich als sehr fruchtbar erwiesen, um die aufgrund der parteipolitischen Hintergründe der Kandidaten erwarteten Unterschiede zwischen den Begriffsverständnissen herauszuarbeiten. Die multimodale Diskursanalyse zeigt, wie sich sprachliche und visuelle diskursive Muster sowie unterschiedliche inhaltliche und rhetorische Bezüge der Kandidaten ergänzen und verstärken können. So trägt der Artikel zur bisher weniger stark ausgeprägten politikwissenschaftlich inspirierten Analyse des Begriffs bei.

Bei Van der Bellen und Hofer stehen sich Heimatbegriffe mit unterschiedlichen wissenschaftstheoretischen Begriffsverständnissen entgegen, die sich vor allem in ihrer Offenheit bzw. Geschlossenheit unterscheiden und unterschiedliche gesellschaftspolitische Modelle repräsentieren. Van der Bellen vertritt einen offenen Heimatbegriff, wie auch Bernhardt und Liebhart (2017) beobachten. Dieser entspricht eher einem konstruktivistischen Begriffsverständnis (Kühne/Spellerberg 20IO). Er präsentiert durch unterschiedlichste interdependente Bezüge mehrere „Heimaten“, die parallel zueinander existieren und sich überlappen können. Entgegen traditionellen Heimatverständnissen (Kühne/Spellerberg 20IO) bezieht er sich nicht nur auf einen Raum und es findet bei Van der Bellen auch keine generelle Verklärung einer spezifischen Vergangenheit oder Zukunft statt. Die postulierten Themen und Werte spiegeln eine Wertschätzung sowohl des Autochthonen als auch des Nicht-Autochthonen wider. Die positive Grundhaltung gegenüber Veränderung betont die Offenheit und Wandelbarkeit dieser "Heimaten“. Van der Bellen versucht das in seinen Augen bisher dominante, enge Verständnis von „Heimat" als geschlossenes, nicht veränderbares Gebilde - wie es beispielsweise von seinem Gegenkandidaten Hofer und der FPÖ vertreten wird - zu dekonstruierten. Zudem betont er insbesondere die sozialen und emotionalen Dimensionen von "Heimat" (Kühne/Schönwald 2015). In den multiplen "Heimaten“ finden sich in den Aussagen Van der Bellens entsprechend multiple Identifikationsangebote sowohl auf individueller als auch kollektiver Ebene, die auf ein nicht-essenzialistisches Identitäts- und Heimatverständnis hinweisen.

Nichtsdestotrotz präsentiert Van der Bellen oftmals ein idealtypisches, ländlich geprägtes Heimatbild durch die gleichzeitige Betonung der räumlichen und symbolischen Dimensionen. Das Kaunertal, die alpenländische Landschaft und der Dialekt bilden besonders zentrale Referenzen, die ein romantisiertes Bild von "Heimat" 
vermitteln. Es erinnert an die Deutung von „Heimat“ als kulturelles Konzept in den I950er Jahren (Bastian I995; Kühne/Spellerberg 20I0). Diese Konnotation kann mit wahlstrategischen Erwägungen in Verbindung gebracht werden. Möglicherweise wollte Van der Bellen so WählerInnen in ländlichen Regionen ansprechen, da es ihm dort im ersten Wahlgang an Zustimmung mangelte (Bundesministerium für Inneres 2016). Die stark regionalisierten, ländlichen Bezüge könnten auch darauf abgezielt haben, den Kandidaten nahbar(er) erscheinen zu lassen, da er als ehemaliger Universitätsprofessor und langjähriger Grünen-Politiker laut Umfragen hier Defizite aufwies (Filzmaier et al. 2017).

Im Vergleich zu Van der Bellen repräsentiert Hofer einen wesentlich exklusiveren, statischeren Heimatbegriff, der sich eher dem positivistischen Begriffsverständnis (Kühne/Spellerberg 20IO) zuordnen lässt. Er bezieht sich in erster Linie auf die nationale Ebene und bedient sich auch rhetorisch und in der Bildsprache einer nationalen Symbolik, beispielsweise durch die starke Präsenz der österreichischen Nationalflagge. Auf diese Weise betont Hofer stark die räumliche Dimension von „Heimat". Zudem hebt er die Notwendigkeit bisherige Leistungen und „unser Österreich“ (VI) bewahren zu müssen hervor. Dieser geschlossene, national-staatsbürgerschaftlich orientierte Heimatbegriff von Hofer entspricht eher dem Bild von Heimat, das bisher häufig von rechten und konservativen PolitikerInnen vermittelt wurde bzw. immer noch wird (Kühne/Spellerberg 20I0; Bastian 1995). Mit dieser Deutung steht er in der Tradition seiner Partei, da die FPÖ den Heimatbegriff traditionell national-republikanisch deutet (Marquart 2013; Preglau 200I). Die Betonung der ÖsterreicherInnen sowie der Grenzen von Veränderung legt eine Höherschätzung des Autochthonen gegenüber dem Allochthonen nahe, die die „Dimension von Ein- und Ausgrenzung "(Kühne/Spellerberg 20IO) im Kontext des Heimatbegriffs in den Vordergrund rückt. Diese Äußerungen lassen auf ein essenzialistisches Verständnis von Identität und Heimat schließen.

Gleichermaßen lässt Hofer durch vage Äußerungen in Kombination mit jenem Essenzialismus Raum für die Interpretationen und das Heimatverständnis der RezipientInnen. Außerdem suggeriert er wiederholt, dass sein Gegenkandidat den Heimatbegriff nur zu Wahlkampfzwecken instrumentalisiert und er selbst bzw. seine Partei diesen Begriff tatsächlich repräsentieren. Auf diese Weise möchte er seine eigene Deutungshoheit über den Heimatbegriff betonen.

Das für diesen Artikel gewählte illustrative Forschungsdesign bietet erste Einblicke in sehr unterschiedliche Verständnisse des Begriffs „Heimat", die sich in politischen und öffentlichen Diskursen in Österreich finden. Jedoch erlaubt die Analyse noch keine Aussagen darüber, inwieweit die Verständnisse der Kandidaten (dominante) Deutungen des Heimatbegriffs in den aktuellen österreichischen Diskursen repräsentieren oder auf die Polarisierung im Bundespräsidentschaftswahlkampf 2016 zurückzuführen sind. Um solche Fragen beantworten zu können, wären insbesondere methodische Vertiefungen der Analyse, wie beispielsweise ein Langzeitvergleich und eine Ausweitung des zugrundeliegenden Datenmaterials, aber auch weiterführende diskurstheoretische Schärfungen denkbar.

\section{Literatur}

Adunka, Evelyn (2002), Exil in der Heimat: Über die Österreicher in Israel, Studien-Verlag: Innsbruck.

Anderson, Benedict (2006), Imagined Communities: Reflections on the Origin and Spread of Nationalism, London: Verso Books.

Barthes, Roland (1988). Einführung in die strukturalen Erzählungen, in: Barthes, Roland (Hg.): Das semiologische Abenteuer, Frankfurt am Main: Suhrkamp, IO2-I 43.

Bastian, Andrea (1995), Der Heimat-Begriff: Eine begriffsgeschichtliche Untersuchung in verschiedenen Funktionsbereichen der deutschen Sprache, Berlin: De Gruyter.

Bernhardt, Petra/Karin Liebhart (20I7), Politik auf Instagram: Bildstrategien von Norbert Hofer und Alexander Van der Bellen im Bundespräsidentschaftswahlkampf 2016, in: SWS Rundschau, Vol. 57(2), I46-167.

Bundesministerium für Inneres (2016), Bundespräsidentenwahl 2016 - I. Wahlgang: Gesamtergebnis inklusive Verlautbarung der Bundeswahlbehörde, Internet: https://www.bmi.gv.at/4I2/Bundespraesidentenwahlen/Bundespraesidentenwahl_20I6/ start.aspx\#pk_05 (Zugriff: 08.II.2019).

Demokratiezentrum Wien (2019), FPÖ-Plakat aus dem Nationalratswahlkampf 2006: Heimat statt Schüssel und Brüssel, Internet: http://www.demokratiezentrum.org/wissen/bilder.html?index=1933 (Zugriff: O8.II.20I9).

Derrida, Jacques (1983), Grammatologie, Frankfurt am Main: Suhrkamp.

Derrida, Jacques (1999), Die Différance, in: Derrida, Jacques (Hg.): Randgänge der Philosophie, Wien: Passagen Verlag, 3I-56.

Dzudzek, Iris (20I6). Kreativpolitik. Über die Machteffekte einer neuen Regierungsform des Städtischen, Bielefeld: Transcript Verlag.

Filzmaier, Peter/Flooh Perlot/Martina Zandonella (2017), Die Bundespräsidentenwahl 2016 in Kärnten, in: Anderwald, Karl/Peter Filzmaier/Karl Hren (Hg.): Kärntner Jahrbuch für Politik 20I6, Klagenfurt: Mohorjeva Hermagoras, 9-33. 
Foucault, Michel (197I), Die Ordnung der Dinge: Eine Archäologie der Humanwissenschaften, Frankfurt am Main: Suhrkamp.

Foucault, Michel (1973), Archäologie des Wissens, Frankfurt am Main: Suhrkamp.

Freiheitliche Partei Österreichs (2019), Freiheitliche Partei Österreichs - Webseite, Internet: https://www.fpoe. at/ (Zugriff: 08.1I.2019).

Gaigg, Vanessa (20I8), SPÖWien setzt künftig auf Heimat, in: derstandard.at, I4.05.2018, Internet: https://www. derstandard.at/jetzt/livebericht/2000079666943/ redcontent/IOooII7I38/michael-ludwig-praesentiert-sein-neues-team-fuer-wien ? responsive $=$ false (Zugriff: o8.II.2019).

Gensheimer, Cynthia Francis/Anton Hieke (2018). Heimat and Home: Mobility Among Jews in Quincy, Illinois, in: American Jewish History, Vol. IO2(2), 255-277.

Glasze, Georg (2008), Vorschläge zur Operationalisierung der Diskurstheorie von Laclau und Mouffe in einer Triangulation von lexikometrischen und interpretativen Methoden, in: Historical Social Research, Vol. 33(I), I85-223.

Glasze, Georg (2014), Politische Räume: Die diskursive Konstitution eines „geokulturellen Raums“ - die Frankophonie, Bielefeld: Transcript Verlag.

Glasze, Georg (2015), Identitäten und Räume als politisch: Die Perspektive der Diskurs- und Hegemonietheorie, in: Europa regional, Vol. 2 I(I-2), 23-35.

Gördesli, M. Melih (20II), Ohne Heimat: Erinnerungen und Anschauungen eines Einwanderers, Aachen: Karin Firscher Verlag.

Huemer, Peter (2006), Heimat, Lügen, Literatur: Texte zur gegenwärtigen Befindlichkeit, Wien: Verlag Der Apfel.

Kühne, Olaf (2OII), Heimat und sozial nachhaltige Landschaftsentwicklung, in: Raumforschung und Raumordnung, Vol. 69(5), 29I-30I.

Kühne, Olaf/Antje Schönwald (2015), Identität, Heimat sowie In- und Exklusion: Aspekte der sozialen Konstruktion von Eigenem und Fremdem als Herausforderung des Migrationszeitalters, in: Nienaber, Birte/ Ursula Roos (Hg.): Internationalisierung der Gesellschaft und die Auswirkungen auf die Raumentwicklung. Beispiele aus Hessen, Rheinland-Pfalz und dem Saarland, Arbeitsberichte der ARL I3, Verlag des ARL: Hannover, IOO-IIO.

Kühne, Olaf/Annette Spellerberg (2010), Heimat in Zeiten erhöhter Flexibilitätsanforderungen: Empirische Studien im Saarland, Wiesbaden: VS Verlag für Sozialwissenschaften.

Laclau, Ernesto (1990), New Reflections on the Revolution of our Time, London: Routledge, Chapman \& Hall.

Laclau, Ernesto/Chantal Mouffe (200I), Hegemony \& Socialist Strategy: Towards a Radical Democratic Politics, London: Verso Books.
Liebhart, Karin/Petra Bernhardt (2017), Political Storytelling on Instagram: Key Aspects of Alexander Van der Bellen's Successful 2016 Presidential Election Campaign, in: Media and Communication, Vol. 5(4), I525 .

Marquart, Franziska (2013), Rechtspopulismus im Wandel: Wahlplakate der FPÖ von 1978-2008, in: Österreichische Zeitschrift für Politikwissenschaft, Vol. 42(4), 353-37I.

Meier, Stefan (2008), Von der Sichtbarkeit im Diskurs: Zur Methode diskursanalytischer Untersuchung multimodaler Kommunikation, in: Warnke, Ingo/ Jürgen Spitzmüller (Hg.): Methoden der Diskurslinguistik: Sprachwissenschaftliche Zugänge zur transtextuellen Ebene, Berlin/New York: De Gruyter, 263-286.

Merkel, Hansi-Christiane (2016), Zwischen Herkunft und Heimat: Zwischen Verlassen und Verlust, in: Leidfaden, Vol. 5(3), 47-53.

Orkowski, Hubert (Hg.) (I993), Heimat und Heimatliteratur in Vergangenheit und Gegenwart, Poznań: Wydawn/New Ton.

Pelinka, Anton (2004), Das politische System Österreichs, in: Ismayr, Wolfgang (Hg.): Die politischen Systeme Westeuropas, Wiesbaden: VS Verlag für Sozialwissenschaften, 52I-552.

Pelinka, Anton/Sieglinde Rosenberger (2007), Österreichische Politik: Grundlagen, Strukturen, Trends, Wien: facultas Verlag.

Preglau, Max (200I), Rechtsextrem oder postmodern? Über Rhetorik, Programmatik, Interaktionsformen und ein Jahr Regierungspolitik der (Haider-)FPÖ, in: SWS-Rundschau, Vol. 4I(2), I93-2I3.

Renggli, Cornerlia (2014), Komplexe Beziehungen beschreiben: Diskursanalytisches Arbeiten mit Bildern, in: Eder, Franz X./Oliver Kühschelm/Christina Linsboth (Hg.): Bilder in historischen Diskursen, Wiesbaden: Springer VS, 45-6I.

Saussure, Ferdinand de (200I), Grundfragen der allgemeinen Sprachwissenschaft, Berlin: De Gruyter.

Sebald, W. G. (I99I), Unheimliche Heimat: Essays zur österreichischen Literatur, Salzburg: Fischer Taschenbuch Verlag.

Steiner, Gertraud (1987), Die Heimat-Macher: Kino in Österreich I946-I966, Wien: Verlag für Gesellschaftskritik.

Stuhlpfarrer, Martin (2018), Wiener SPÖ: Die „Heimat“ wird rot, in: diepresse.com, 03.05.2018, Internet: https:/diepresse.com/home/panorama/wien/5416202/ Wiener-SPOe_Die-Heimat-wird-rot (Zugriff: O8.II. 20I9).

Thaler, Walter (20I3), Der Heimat treue Hasser. Schriftsteller und Politik in Österreich ; ein politisches Lesebuch, Wien: nap - new academic press. 
Wimmer, Adi (Hg.) (1993), Die Heimat wurde ihnen fremd, die Fremde nicht zur Heimat. Erinnerungen österreichischer Juden aus dem Exil, Wien: Verlag für Gesellschaftskritik.

Yanow, Dvora (1996), How does a policy mean? Interpreting policy and organizational actions, Washington, D.C.: Georgetown University Press.

\section{Autorin}

Andrea Tony Hermann ist wissenschaftliche Mitarbeiterin am Institut für Strategieanalysen und an der Universität Graz. Ihre Arbeits- und Forschungsschwerpunkte umfassen politische Kulturen, die Rolle von wissenschaftlicher und nicht-wissenschaftlicher Expertise in politischen Prozessen und in den Medien, Regionalpolitik und -entwicklung in ländlichen Räumen sowie Umwelt- und Klimapolitik. 
